\title{
Transformaciones en las prácticas educativas en contextos de COVID-19. Percepciones de un grupo de docentes argentinos
}

Transformations in educational practices in COVID-19 contexts.

Perceptions of a group of Argentinean teachers 


\title{
Transformaciones en las prácticas educativas en contextos de COVID-19. Percepciones de un grupo de docentes argentinos
}

Transformations in educational practices in COVID-19 contexts. Perceptions of a group of Argentinean teachers

\author{
Romina Cecilia Elisondo
}

\begin{abstract}
Resumen: En el contexto de emergencia mundial y suspensión de actividades presenciales en las instituciones educativas, el objetivo de este estudio fue analizar los cambios en las prácticas docentes en diferentes momentos del aislamiento y distanciamiento social por COVID-19. Se realizó un estudio cualitativo fenomenológico con el objetivo de comprender sentidos y significados sobre prácticas educativas. El estudio se desarrolló entre agosto y septiembre de 2020 dando continuidad a una investigación anterior que se realizó en los primeros momentos del aislamiento social. Participaron del presente estudio 30 docentes que residen en el Departamento de Río Cuarto (Argentina). Todas las personas participantes del estudio son mujeres. El muestreo fue intencional y por oportunidad. Para la recolección de datos se utilizaron cuestionarios con preguntas de opción abierta y entrevistas semiestructuradas. Se realizaron procesos de triangulación y validación comunicativa. A partir del análisis cualitativo con ATLAS.ti (segmentación, construcción de citas, codificación, creación de memos, categorización y teorización), se construyeron cinco categorías generales para sistematizar los datos: Trencadís educativo en cuarentena; Brechas y desigualdades profundas; Sobrecarga docente $y$ trabajo colaborativo Especificidades de las propuestas según niveles educativos y Aprendizajes inesperados. Los resultados indican que el profesorado ha creado diversas propuestas educativas utilizando diferentes mediaciones $y$ generando cambios en las prácticas. Las participantes perciben sobrecarga en sus actividades y desigualdades educativas. Los análisis también muestran especificidades según niveles y aprendizajes inesperados en docentes y estudiantes. Desde las teorías de la creatividad, se observa que muchas de las propuestas pedagógicas relatadas incluyen componentes que han sido definidos como contextos creativos de enseñanza y aprendizaje. Pensar en educación aumentada y multimodal, que integre diferentes prácticas comunicativas, es interesante para transitar en las nuevas normalidades. Las particularidades de los contextos creativos pueden ser de relevancia para discutir futuros entornos multimodales de educación. Asimismo, se considera indispensable revalorizar las experiencias educativas construidas en pandemia y reconocer el valor del trabajo realizado por el profesorado, el alumnado y las familias.
\end{abstract}

Palabras clave: creatividad, enseñanza, COVID-19, desigualdad

\begin{abstract}
In the context of a global emergency and suspension of face-to-face activities in educational institutions, the objective of the study is to analyze changes in teaching practices at different times of isolation and social distancing due to COVID-19. A qualitative phenomenological study was carried out in order to understand meanings and meanings about educational practices. The study was carried out between August and September 2020, continuing a previous investigation that was carried out in the first moments of social isolation. Thirty teachers residing in the Department of Rio Cuarto (Argentina) participated in the present study. All the people participating in the study are women. The sampling was intentional and by opportunity. For data collection, it will be used in questionnaires with open-choice questions and semistructured interviews. Triangulation and communicative validation processes were carried out. From the qualitative analysis with ATLAS.ti (segmentation, construction of citations, coding, creation of memos, categorization and theorization), five general categories were constructed to systematize the data: Trencadís educational in quarantine; Deep gaps and inequalities; Teaching overload and collaborative work Specificities of the proposals according to educational levels and Unexpected learning. The results indicate that teachers have created various educational proposals, using different mediations and generating changes in practices. The participants perceive overload in their activities and educational inequalities. The analyzes also show specificities according to levels and unexpected learning in teachers and students. From the theories of creativity, it is observed that many of the related pedagogical proposals include components that have been defined as creative teaching and learning contexts. Thinking about augmented and multimodal education, which integrates different communicative practices, is interesting to move into the new normalities. The particularities of creative contexts may be relevant to discuss future multimodal educational environments. Likewise, it is considered essential to revalue the educational experiences built in a pandemic and to recognize the value of the work done by teachers, students and families.
\end{abstract}

Keywords: creativity, teaching, COVID-19, inequality

1 Investigadora Adjunta del Consejo Nacional de Investigaciones Científicas y Técnicas, Buenos
Aires, Argentina. Profesora Adjunta en la Universidad Nacional de Río Cuarto, Córdoba, Argentina.
Doctora en Psicología (España), Master en Educación. Dirección electrónica: relisondo@hum.unrc.edu.ar Orcid: https://orcid.org/0000-0002-7841-9878

Artículo recibido: 28 de noviembre, 2020

Enviado a corrección: 5 de marzo, 2021

Aprobado: 28 de junio, 2021 


\section{Introducción}

El 2020 fue un año de cambios en la vida de la mayoría de los habitantes del planeta. Millones de personas fueron aisladas en sus hogares, muchas debieron dejar de trabajar o modificar sus modalidades laborales, las instituciones educativas suspendieron sus actividades presenciales, solo por mencionar algunas transformaciones en la vida cotidiana. Según la UNESCO (2020a), a nivel mundial, la suspensión de las actividades educativas afectó aproximadamente al 90\% del estudiantado. Más de 1500 millones de personas han visto alterados sus procesos de aprendizaje en instituciones educativas formales. Ante la emergencia sanitaria y el cierre de las instituciones educativas, docentes y directivos han generado diversas estrategias para sostener la continuidad educativa en los diferentes niveles del sistema. En muchos casos, las propuestas se elaboraron de forma repentina, con escasa programación, y sin considerar los recursos tecnológicos que tenían docentes y estudiantes en sus hogares.

A partir del 16 de marzo de 2020, en Argentina se suspendió por decreto el dictado de clases presenciales en los niveles inicial, primario, secundario y superior en todas sus modalidades. Datos de la UNESCO (2020a) indican que más de 14 millones de estudiantes argentinos se han visto afectados por la suspensión de clases presenciales. Según los datos de la encuesta a docentes desarrollada por el Ministerio de Educación de Argentina (2020), todas las escuelas llevaron adelante propuestas de continuidad pedagógica, y el 95\% de los hogares recibió propuestas pedagógicas durante todo el período de aislamiento social, preventivo y obligatorio. En la Encuesta Rápida COVID-19 de UNICEF (2020), realizada en Argentina, se sostiene que el acceso a recursos tecnológicos es un factor muy relevante al momento de analizar la situación escolar de los niños, niñas y adolescentes, dado que en el contexto actual se tornan fundamentales para sostener la comunicación educativa. Los resultados muestran que el $18 \%$ de los y las adolescentes no cuenta con acceso a Internet en sus hogares, Asimismo, el $37 \%$ de las personas participantes afirma que no dispone de una computadora o tableta para realizar sus tareas escolares.

Tal como revelan los datos de las encuestas y estudios en diferentes contextos y niveles (Bocchio, 2020; Lion, 2020; Ruiz, 2020; Tranier, Bazán, Porta y Di Franco, 2020), una de las problemáticas más preocupantes en el contexto argentino, pero también de muchos otros países, es la desigualdad educativa que se genera por la carencia de conectividad y recursos tecnológicos de amplios sectores de la sociedad. Asimismo, la disponibilidad de conocimientos previos y competencias digitales también genera brechas entre diferentes sectores, tanto en 
la población estudiantil como en el profesorado. Álvarez, Gardyn, lardelevsky y Rebello (2020) consideran que las brechas en el acceso y uso de recursos digitales profundizan la segmentación educativa en pandemia. Los resultados indican que, en las instituciones con población vulnerable, las acciones prioritarias son la alimentación, el mantenimiento del vínculo y la construcción de redes para evitar el abandono. En el contexto universitario, los estudios de Maldonado, Miró, Stratta, Barreda y Zingaretti (2020) y Macchiarola, Pizzolitto, Solivellas y Muñoz (2020) también señalan dificultades en el acceso al material, la conectividad y la disponibilidad de recursos tecnológicos.

En este contexto de emergencia mundial y suspensión de actividades presenciales en las instituciones educativas, se desarrolla el presente estudio con el objetivo de analizar cambios en las prácticas docentes en diferentes momentos del aislamiento y distanciamiento social. Interesa recuperar, desde las voces de un grupo de docentes, transformaciones en sus prácticas y motivos que originan estos cambios. Igualmente, es objetivo del estudio comprender los significados construidos por el profesorado respecto de las acciones e interacciones desarrolladas, las emociones emergentes y los desafíos pendientes. Es relevante también analizar la especificidad de las prácticas y transformaciones según los diversos niveles educativos.

El estudio se desarrolló entre agosto y septiembre de 2020 dando continuidad a una investigación anterior que se desarrolló en los primeros momentos del aislamiento social (Elisondo, Melgar, Chesta y Siracusa, 2020). En dicho estudio participaron 140 docentes que residen en el Departamento de Río Cuarto (Córdoba) y trabajan en diferentes niveles y modalidades del sistema educativo. Los resultados de aquel estudio indicaron que el profesorado construye prácticas creativas que permiten sostener el compromiso con la educación en contextos inciertos y desiguales. Las personas participantes de la investigación perciben desigualdades tanto en la disponibilidad de recursos materiales como simbólicos. Asimismo, se observa que la superposición de actividades laborales, personales, domésticas y de cuidado caracteriza la vida cotidiana del profesorado.

El propósito de la presente investigación es producir conocimientos respecto de prácticas educativas desarrolladas en pandemia, recuperando las voces de un grupo de docentes. Estos conocimientos podrán contribuir en el diseño y la ejecución de proyectos y políticas educativas de continuidad pedagógica en pandemia y post-pandemia. Para pensar alternativas educativas en tiempos inciertos, es indispensable escuchar las voces del profesorado protagonistas en el sostenimiento del derecho a la educación. Esperamos realizar aportes al diseño de planes y 
programas educativos, recuperando prácticas creativas desarrolladas en contextos de educación remota por emergencia sanitaria.

\section{Referente teórico}

El presente estudio se sustenta en desarrollos actuales del campo de la creatividad en contextos educativos (Gajda, Beghetto y Karwowski, 2017; Richardson y Mishra, 2018). Se define la creatividad como un proceso cognitivo socio-cultural que posibilita el desarrollo de acciones y productos novedosos, originales y relevantes en diferentes contextos. En tanto proceso cognitivo, supone la puesta en juego de pensamientos divergentes para la resolución de problemas buscando respuestas originales. Como proceso sociocultural, implica interacciones con otras personas y con objetos de la cultura para la creación de nuevos productos y prácticas alternativas (Glaveanu, et al. 2019).

Siguiendo los planteamientos de Cristiano (2018), se enfatiza el papel de los actores sociales en el desarrollo de acciones creativas, es decir prácticas sociales divergentes, originales y alternativas. Consideramos la creatividad como una potencialidad que pueden desarrollar las personas en diferentes situaciones de enseñanza y aprendizaje. Beghetto y Karwowski (2018) destacan la importancia de reconocer la enseñanza para la creatividad y el aprendizaje creativo como acciones dinámicas y activas que dependen de una amplia gama de creencias personales interrelacionadas (autoconcepto, autoeficacia e identidad creativa). Según los autores estas creencias desempeñan funciones reguladoras claves en la creatividad solo si estudiantes y docentes perciben el valor y el sentido de los conocimientos y las actividades propuestas, y creen que son capaces de hacer contribuciones originales, podrán lograr acciones creativas.

Investigaciones actuales (Davies et al. 2013; Gajda et al. 2017; Richardson y Mishra, 2018) indican que las siguientes acciones docentes son propicias para la creatividad:

- creación de espacios para la imaginación y el juego

- promoción de la autonomía, el pensamiento flexible, la autoconfianza y el compromiso

- generación en propuestas dialógicas que permitan expresar y explorar ideas divergentes

- potenciación de atmósferas de cooperación donde los estudiantes puedan tomar riesgos, cometer errores y plantear ideas alternativas.

- diseño actividades fuera de las aulas

- desarrollo de tareas de final abierto y resolución divergentes 
Los planteamientos sobre creatividad y educación desarrollados anteriormente son previos a la crisis mundial por la pandemia. Es relevante resignificar estas consideraciones atendiendo la complejidad de las condiciones sanitarias, económicas y educativas actuales. Se destacan los aportes de Berg, Blum Vestena y Costa-Lobo (2020) que reconocen el papel de la creatividad como herramienta para la transformación educativa en tiempos de pandemia. Los autores invitan a investigar respecto de las consecuencias educativas de la pandemia para producir conocimientos que aporten a la construcción de sistemas educativos sólidos, cooperativos y críticos que promuevan la libertad y el desarrollo social.

Con base en estudios teóricos, investigaciones empíricas y experiencias educativas, en Elisondo (2015) se desarrollan cinco ideas para pensar los contextos de enseñanza y aprendizaje desde una perspectiva creativa: aumentar, invertir, investir , abrir la educación y generar indisciplina óptima. Estas ideas adquieren una significatividad especial en el contexto actual de pandemia. En la emergencia sanitaria y educativa se observan prácticas que se corresponden con las ideas esbozadas en aquel momento, por ejemplo, propuestas de educación aumentada a partir de mediaciones tecnológicas, clases invertidas, experiencias con docentes inesperados y visitas virtuales a museos, conferencias y eventos. También se visualizan propuestas educativas que intentan sostener la motivación y el vínculo intersubjetivo (llamadas, mensajes, audios y videos motivadores). Asimismo, muchas de las prácticas han transgredido normas y generado rupturas con formas habituales de enseñar y aprender.

En el complejo contexto social actual y según los planteos teóricos mencionados, nos proponemos analizar, desde las voces del profesorado, acciones desarrolladas durante la pandemia, cambios y transformaciones emergentes y posibilidades futuras. La presente investigación pretende comprender el dinamismo de los procesos educativos en el contexto actual según las percepciones y valoraciones docentes. Es nuestro interés comprender las prácticas de los docentes basándonos en teorías actuales de la creatividad en contextos educativos, resignificando conceptos y estudios anteriores, a la luz de la complejidad que genera la pandemia. Nos proponemos generar conocimientos que, de alguna manera, aporten al diseño de nuevas acciones y políticas educativas de cara a las nuevas normalidades que se avecinan. 


\section{Metodología}

\subsection{Enfoque}

En el marco de un enfoque cualitativo de investigación, se realizó un estudio fenomenológico (Creswell y Poth, 2007) con el objetivo de comprender sentidos y significados construidos respecto de prácticas educativas en tiempos de COVID-19.

\subsection{Unidades de análisis}

Participaron 30 docentes que residen en el Departamento de Río Cuarto, Córdoba, Argentina. Todas las personas participantes del estudio son mujeres. La edad de las participantes oscila entre 25 y 55 años. En la muestra se incluyeron docentes de diferentes niveles del sistema educativo: Inicial $(n=6)$, Primario $(n=7)$, Secundario $(n=7)$ y Superior $(n=10)$. Las participantes manifestaron tener una antigüedad en el cargo docente de entre 5 y 10 años. Las docentes se han formado académicamente en instituciones de educación superior de Córdoba, cinco $(n=5)$ han realizado estudios de posgrado.

La muestra se seleccionó, de manera intencional y por conveniencia, de un estudio anterior (Elisondo, Melgar, Chesta y Siracusa, 2020), las 30 personas que forman parte de la muestra indicaron su deseo de participar de esta segunda etapa de la investigación. La selección de la muestra se realizó teniendo en cuenta los objetivos de la investigación: analizar procesos y cambios en las prácticas educativas en tiempos de COVID-19. En este sentido, cobra relevancia incluir en la muestra a personas participantes del estudio realizado en el inicio del aislamiento social (abril de 2020), con el fin de analizar transformaciones en la prácticas.

Las participantes fueron informadas respecto de los objetivos y alcances de la investigación. También se les comunicó sobre la confidencialidad de los datos y las estrategias de preservación del anonimato. Las participantes dieron el consentimiento para la realización del estudio y la publicación de resultados.

\subsection{Técnicas de recolección}

El estudio se desarrolló entre agosto y septiembre de 2020. Conforme lo manifestado por las participantes, según su interés y disponibilidad de tiempo, se utilizaron dos instrumentos de recolección de datos: un cuestionario con preguntas de opción abierta administrado en GoogleForms y entrevistas semiestructuradas a través de Google Meet. En ambos instrumentos, las preguntas giraron en torno a cambios en los procesos educativos en diferentes momentos de aislamiento y distanciamiento social por COVID-19. Asimismo, 
indagamos respecto de estrategias pedagógicas, interacciones con estudiantes, prácticas novedosas y dificultades emergentes. También, se consultó a las docentes respecto de los aprendizajes logrados por el alumnado y las modalidades de evaluación implementadas (Ver Anexo 1).

\subsection{Procesamiento de análisis}

Se utilizó el programa ATLAS.Ti8 para el análisis cualitativo. Se realizaron procedimientos segmentación, construcción de citas, codificación (construcción, fusión, eliminación), creación de memos, categorización y teorización (comparación, contrastación, ordenación, establecimiento de relaciones y jerarquización). A partir de dichos procedimientos, se construyeron cinco categorías generales para sistematizar los datos (Ver Tabla 1). En cada categoría emergente se analizan las evidencias conforme a teorías actuales de la creatividad y estudios preliminares de prácticas educativas en tiempos de COVID-19. Para evitar sesgos de interpretación y evaluar la calidad del proceso, se triangularon análisis entre diferentes investigadores. También se llevó a cabo un proceso de validación comunicativa solicitando a un grupo de participantes la lectura crítica de las categorías, las evidencias y los análisis. Los investigadores y las participantes acordaron que los análisis realizados guardan congruencia con las expresiones recogidas de los cuestionarios y las entrevistas.

Tabla 1: Descripción de las categorías de análisis del estudio. Córdoba-Argentina, 2020.

\begin{tabular}{|l|l|}
\hline \multicolumn{1}{|c|}{ Categorías } & \multicolumn{1}{c|}{ Descripción } \\
\hline Trencadís educativo en cuarentena & $\begin{array}{l}\text { Transformaciones en las prácticas educativas. } \\
\text { Actividades de enseñanza y de aprendizaje. El } \\
\text { papel de las tecnologías. Procesos de cambio } \\
\text { en diferentes momentos del aislamiento y el } \\
\text { distanciamiento. }\end{array}$ \\
\hline Brechas y desigualdades profundas & $\begin{array}{l}\text { Desigualdades sociales y educativas. Brecha } \\
\text { digital. Acceso a recursos tecnológicos. } \\
\text { Conectividad. Condiciones materiales y } \\
\text { simbólicas. }\end{array}$ \\
\hline Sobrecarga docente y trabajo colaborativo & $\begin{array}{l}\text { Transformaciones en el trabajo docente. } \\
\text { Sobrecarga laboral. Prácticas colaborativas } \\
\text { entre docentes . Emociones }\end{array}$ \\
\hline Especificidades de las propuestas según \\
niveles educativos & $\begin{array}{l}\text { Particularidades de las prácticas docentes en } \\
\text { nivel inicial, primario, secundario y superior. } \\
\text { Similitudes y diferencias. }\end{array}$ \\
\hline Aprendizajes inesperados & $\begin{array}{l}\text { Aprendizajes no planificados construidos por } \\
\text { docentes y estudiantes. Apropiación de } \\
\text { recursos tecnológicos. Prácticas solidarias y } \\
\text { resilientes. }\end{array}$ \\
\hline
\end{tabular}

Fuente: elaboración propia 


\section{Resultados}

\subsection{Trencadís educativo en cuarentena}

La metáfora de la práctica educativa como trencadís ${ }^{4}$ parece reflejar el trabajo de las docentes en tiempos de pandemia. Las expresiones de las participantes muestran prácticas artesanales, con diferentes colores y formas, que articulan una trama compleja. La búsqueda de piezas que encajen adecuadamente, los colores necesarios y las formas justas, describe, en parte, el importante esfuerzo de las docentes en la construcción de estrategias de enseñanza en contextos inciertos, desiguales y difíciles. Las docentes, en diferentes momentos de la pandemia, han modificado sus prácticas a partir de autorregulaciones y reflexiones sobre los aprendizajes y las posibilidades del alumnado. Las narrativas muestran con claridad el dinamismo de las planificaciones y las propuestas educativas en diferentes momentos del aislamiento. Mientras que un primer momento, predomina la improvisación y la prueba de alternativas, luego las prácticas se redefinen y ajustan a las posibilidades y realidades del estudiantado y sus familias. Sabulsky (2020) también se refiere a un primer momento de ensayo-error que debería dar paso a la planificación crítica, reflexiva y consensuada, en el marco de programas institucionales y políticas educativas más amplias. Compartimos a continuación algunos fragmentos de las expresiones de las docentes, que ilustran las categorías construidas (estos fragmentos muestran solo algunos segmentos del corpus analizado):

Los principales cambios que observo es que se volvió más personalizada y vinculante, el ritmo de los contenidos a dar lo marcan los estudiantes y además requiere de un mayor esfuerzo como docente en amplios sentidos (Docente de nivel secundario).

Los cambios son muy grandes, no tener contacto directo con los niños/as dificulta la tarea. Trabajo en una escuela rural, en donde no tiene mucho Internet, solo datos móviles, por ende el contacto que se tiene con los niños/as es a través de algunos vídeos, $y$ audios. $Y$ depende en gran medida del vínculo que se tiene con la familia, son ellos quienes realizan de puente entre nosotros y los/as estudiantes (Docente de nivel inicial).

Si bien la práctica docente no es rígida y siempre debe ser revisada, en estos tiempos de aislamiento todo va evaluándose en función de prueba y error. La diferencia es que en este momento es más dificultoso por que se depende de otros, y de estar conectados.

\footnotetext{
${ }^{4}$ Aplicación ornamental del mosaico a partir de fragmentos cerámicos unidos con argamasa.
} 
Hay emergentes que no dependen de nuestra práctica y que deben tenerse en cuenta (Docente de nivel inicial).

Las propuestas educativas en tiempos de pandemia se configurarían de una manera más personalizada, no hay una única propuesta, sino múltiples formas y colores (volviendo a la metáfora del trencadís). Estas múltiples propuestas conforman, a la vez, una figura compleja y única: la práctica docente. Esta práctica se construye y reconstruye a partir de múltiples acciones creativas, es decir acciones novedosas que rompen con lo tradicional. En este sentido, y recuperando los aportes de Cristiano (2018), definimos estas prácticas como acciones creativas en tanto propuestas nuevas que generan transformaciones en las prácticas habituales. Las múltiples alternativas que se habilitan también dan cuenta de procesos divergentes de enseñanza. Es decir, las docentes construyen tareas originales para resolver problemas vinculados a la escasa disponibilidad de recursos, competencias previas, ayudas pedagógicas y conectividad. En esta multiplicidad de propuestas y también de diversas formas de resolución es donde observamos prácticas creativas que rompen con las planificaciones y secuencias únicas. Tal como se ha señalado en estudios anteriores (Davies et al. 2013; Gajda et al. 2017; Richardson y Mishra, 2018), la enseñanza creativa parece definirse precisamente por estos rasgos: actividades divergentes, atención a la diversidad, multiplicidad de recursos disponibles y formas alternativas de resolución de problemas de enseñanza y de aprendizaje.

La enseñanza creativa también se caracteriza por cierta improvisación disciplinada (Beghetto y Kaufman, 2011), es decir, supone programación, pero también intersticios para la novedad y lo inesperado. En los análisis realizados, las expresiones de las docentes dan cuenta de prácticas creativas de enseñanza, por ejemplo, se incluyen, en las actividades, personas, animales y objetos disponibles en el hogar, se promueven procesos de resolución de problemas mediante experimentación, se habilitan espacios heterogéneos de comunicación, se ofrecen recursos variados (fotocopias, audios, videos, fotos y textos), entre otras estrategias novedosas. En las expresiones, se observa flexibilidad en la planificación, reflexión permanente sobre la práctica, apertura a la promoción de aprendizajes a partir de experiencias diversas (experimentación, lectura, investigación y viajes virtuales). Encontramos algunas prácticas que podrían definirse didácticas de autor (Porta, 2015), planificaciones únicas y dinámicas que potencian prácticas creativas de enseñanza y de aprendizaje, que dan lugar a participaciones y emociones diversas y propician resoluciones originales. La flexibilidad, la apertura, la reflexión permanente sobre la práctica, el dinamismo y la promoción 
de la divergencia también han sido rasgos definidos como propios de la enseñanza para la creatividad (Jeffrey y Craft, 2004).

Muchas se han propuesto desde cada sala. Actividades lúdicas, de exploración con cuerpos y figuras geométricas... actividades desde las ciencias naturales con experimentación, con propuestas muy interesantes, acercamiento a la palabra escrita proporcionando acceso a diversos formatos textuales en papel y de manera digital, rincón de registros de la investigación, entre muchas otras. Hemos hecho redes virtuales con el Parque Ecológico de la ciudad, con el Municipio en una brigada que cuida el río, con un trabajador del Mercado de Abasto papá de nuestra comunidad escolar, con un médico veterinario (Docente de Nivel Inicial).

Rompecabezas digitales, roscos de preguntas, Padlet, aprender usar Classroom, participar en blog institucional, enviar explicaciones a través de audios e imágenes, clases virtuales por Meet y vídeo llamadas por WhatsApp (Docente de nivel primario).

Considero que las actividades más novedosas tiene que ver con las de experimentación y la creatividad de utilizar los recursos que tengan en casa, cómo realizar circuitos con los materiales que encuentren, realizar pistas de autos, trabajar la lecto escritura a través de la cocina y recetas, experimentar con germinadores y la realización de compost, entre otros. (Docente de Nivel Inicial).

Indiscutiblemente, las tecnológicas juegan un papel muy importante en los procesos educativos en tiempos de COVID-19. La mayoría de las propuestas educativas se han desarrollado a partir de mediaciones tecnológicas en redes (WhatsApp, Facebook, Instagram), plataformas (EDMODO, Classroom, plataformas institucionales) y aplicaciones para la comunicación sincrónica (ZOOM, MEET, etc.). Al igual que en estudios anteriores (Expósito y Marsollier, 2020) y en la encuesta realizada por el Ministerio de Educación (2020), observamos que WhatsApp es el principal medio de comunicación entre docentes, estudiantes y familias.

Se ha utilizado diferentes recursos para enviar las actividades a las familias, se envían por WhatsApp, en forma de texto, en algunas oportunidades cada actividad las he grabado, con un audio hablándoles directamente a los niños/as con diferentes imágenes, en otras ocasiones me he grabado en vídeos explicando la diferentes situaciones a realizar; en un caso que el padre no sabe leer, se envían las actividades en audios. Los vídeos son grabados en un programa con un formato que permita enviarlo por WhatsApp. 
Otra de las modificaciones que se ha realizado, es trata de enviar audios a los niños/as para lograr un contacto directo con ellos/as, en algunas ocasiones se pide también que contesten algunas de las actividades a través de audios o vídeos, a partir de los cuales se puede observar mejor el proceso (Docente de nivel Inicial).

Ahora entregaron infografías digitales y las producciones fueron realmente increíbles!!! Trabajaron con textos de Galeano, Cortazar, Lispector... eso para Ciudadanía y Política y en Historia realizaron infografías de distintos procesos históricos. Y lo segundo, la realizaron de alguna actividad teórica explicada mediante el uso de Screencastify. Generalmente utilizo ese recurso para explicar cada nueva actividad, o dar una mini clase y así los estudiantes disponen de eso de manera diacrónica. Y solicitar que ellos utilicen el mismo recurso para ver cómo resuelven distintas consignas, fue genial y novedoso para mí (Docente de nivel secundario).

Tal como señala Sabulsky (2020) y se corrobora en los datos de la encuesta a docentes del Ministerio de Educación (2020), se observa en las prácticas educativas un bricolaje que integra diferentes tecnologías y también procesos de reedición y adaptación de materiales y recursos por parte de las personas docentes.

Muchos de estos intercambios tienen que ver con propósitos didácticos, aunque no sean del todo claros ni explícitos, y dan cuenta de interesantes esfuerzos por pensar cómo enseñar aun a la distancia. Una apuesta en términos creativos y profesionales por generar el más hermoso bricolaje digital (...) El desbaratamiento del espacio físico, el desdibujamiento de las fronteras que marcaban tanto el adentro como el afuera y el tiempo de la escuela están poniendo en jaque la agenda clásica de la didáctica (Sabulsky, 2020 pp. 38-39)

En suma, el trencadís educativo en tiempo de pandemia se construye y reconstruye a partir de interacciones entre docentes y estudiantes. En estas interacciones el papel de las tecnologías es innegable, tanto en la generación de posibilidades de comunicación así como también, en la mediación de los procesos educativos y las tareas de aprendizaje. Las tecnológicas son un canal de comunicación, pero también un objeto de enseñanza y aprendizaje en sí mismo, no solo son un recurso externo a los sujetos, sino que son artefactos de la cultura que adquieren significatividad y sentido en el marco de prácticas culturales. 
Es relevante señalar que no todas las prácticas educativas se configuran como trencadís creativos, algunas solo reproducen prácticas tradicionales y poco significativas. No todas las prácticas son flexibles y dinámicas conforme a las particularidades de los interlocutores, muchas desconocen a los otros, sus posibilidades y los andamios que disponen. Sin embargo, en el presente estudio interesa recuperar aquellas prácticas que se basan en el reconocimiento del otro, que promueven aprendizajes conceptuales, procedimentales y actitudinales novedosos, y que buscan nuevas formas de combinar formas y colores para sostener los procesos de enseñanza y aprendizaje. De igual modo, es indispensable visibilizar las desigualdades educativas y tecnológicas que se han profundizado en la pandemia. La conectividad y la disponibilidad de recursos tecnológicos además de posibilidades educativas han generado desigualdades y dificultades que serán consideradas en la siguiente categoría de análisis.

\subsection{Brechas y desigualdades profundas}

La pandemia muestra, quizás con más crudeza, las desigualdades sociales, económicas y educativas que caracterizan a la sociedad argentina. Las docentes identifican dificultades económicas, de conectividad y de acceso a los recursos tecnológicos y a las ayudas pedagógicas necesarias. Tal como señalan las docentes estas condiciones desiguales condicionan los procesos educativos de muchos estudiantes y hacen que la continuidad pedagógica sea más difícil.

Las desigualdades sociales tienen una gran incidencia en la continuidad pedagógica, desde al material que se accede, a la diversidad de este material, la posibilidad de algún tipo de contacto, el acompañamiento... (Docente de nivel secundario)

Creo que es una realidad sobre la cual la pandemia echó más luz aún. Las desigualdades siempre estuvieron presentes en nuestro país, pero generalmente dentro del aula todos estábamos "en la misma". Debido al distanciamiento social cada casa se convirtió en un aula y allí muchas veces la continuidad pedagógica se debilita, por multiplicidad de causas. Por lo que creo que cuando llegue el momento de volver estas cuestiones deberán de ser consideradas (Docente de nivel superior)

Las desigualdades no solo caracterizan al estudiantado, sino que también se percibe entre el profesorado. Las participantes indican que muchos docentes no tienen los recursos necesarios para la educación remota. Las participantes también señalan problemáticas 
vinculadas a la organización del tiempo y del espacio en el hogar para el desarrollo de las actividades de enseñanza. Sin embargo, las docentes también señalan acciones institucionales y comunitarias orientadas a atender las problemáticas señaladas.

Es así, en esta pandemia queda totalmente al desnudo las desigualdades sociales, en este escenario, surgen problemáticas sociales muy sensibles y que se reflejan al interior de cada hogar; por tal motivo, como escuela tenemos que ser muy cuidadosas y respetuosas al respecto, no abrumar, contener y ser sensibles a cada realidad. Más que nunca asumir "una pedagogía de la ternura" (Docente de nivel inicial).

En nuestra comunidad la gran mayoría no tiene acceso ni siquiera para usar el WhatsApp, pero hemos ido hasta los hogares concertado encuentros para intentar que no se pierda la continuidad pedagógica, aunque hay situaciones que escapan a nosotros y que no podemos controlar. Se torna más difícil, estando en el aula las tres horas son significativas (Docente de nivel inicial).

Trabajo en una institución en que toda su comunidad es vulnerable y la escuela se acercó a las familias, entregó material didáctico para que los estudiantes no queden fuera del sistema, incluso se les entregó en sus hogares las viandas, a aquellos que ni siquiera podían ir a retirarlas. Los docentes hicimos colectas de alimentos en varias oportunidades y la gestión se encargó de entregarlas a las familias de la escuela que estaban en pésimas condiciones, dado a la falta de trabajo. Hemos estado presentes y el vínculo pedagógico seguimos insistiendo que esté presente en cada estudiante, que nadie quede fuera de la escuela (Docente de nivel secundario).

Como nuestra ciudad es chica podemos llegar absolutamente a todos los estudiantes pero tendimos redes con todos los actores que puedan involucrarse desde el municipio, familias, cooperativa para obtener chip con datos gratis, sociedad rural con una campaña para conseguir celulares y/o computadoras. Ningún alumno se quedó sin las actividades (Docente de nivel primario y secundario).

Tal como ya se había observado en investigaciones realizadas en otros contextos (Bocchio, 2020; Lion, 2020; Ruiz, 2020; Tranier et al. 2020) las dificultades sociales, económicas y educativas se profundizan en la pandemia generando contextos desiguales para estudiantes y docentes. En algunas instituciones, en concordancia con los resultados observados por Álvarez et al. (2020), las prácticas institucionales están orientadas a sostener el vínculo y atender necesidades básicas como la alimentación. Lo educativo pasa a un 
segundo plano ante la necesidad de demandas más urgentes, situación que profundiza aún más las desigualdades. En el estudio de Ardini, Herrera, González Angeletti y Secco (2020) participaron 333 docentes de diferentes niveles educativos de instituciones de la provincia de Córdoba, entre los resultados se destaca la preocupación por construir y reconstruir vínculos pedagógicos, especialmente con estudiantes pertenecientes a sectores vulnerables. Algunos docentes manifestaron "la sensación de correr una carrera contra las desigualdades de origen y el desafío de no profundizarlas en el encuentro digital” (p. 42).

Según Sabulsky (2020), la pandemia muestra con total crueldad la desigualdad social en relación con el acceso a dispositivos, conectividad y condiciones (materiales y simbólicas) para aprender en los hogares. La autora sostiene que la brecha digital no solo se vincula con la disponibilidad de una computadora, sino también con la conectividad, la calidad de los equipos y los recursos simbólicos para el uso de tecnologías con fines académicos. Esta brecha genera condiciones de trabajo pedagógico significativamente diferentes. Asimismo, Expósito y Marsollier (2020) señalan que la situación de pandemia puso en evidencia la desigualdad de oportunidades educativas entre instituciones públicas y privadas, las diferencias entre quienes tuvieron mejor acceso a los recursos tecnológicos y las diferencias en el capital cultural de las familias.

Asimismo, también es importante destacar aquellas prácticas desarrolladas por las instituciones para sostener la continuidad pedagógica: llevar alimentos y tareas a los hogares, conseguir chips, hacer colectas, generar espacios de diálogo y contención, entre tantas otras. En este caso, la creatividad es puesta en juego para sostener el derecho a la educación, no se trata estrictamente de prácticas creativas de enseñanza, sino de acciones indispensables para atender demandas urgentes de estudiantes y docentes. Otros estudios (Bocchio, 2020; Tejero y Tejero, 2020) también han subrayado la importancia de las prácticas solidarias y resilientes desarrolladas por docentes e instituciones en contexto de emergencia sanitaria y educativa por COVID-19. En este sentido, los planteamientos de Tranier et al. (2020) nos interpelan a pensar la crisis actual como posibilidad para la construcción de redes comunitarias solidarias. En la misma línea, Berg et al. (2020) proponen a la creatividad y la autonomía como herramientas de una nueva pedagogía social post-pandemia, que contribuya a la creación de sistemas educativos sólidos, cooperativos y críticos. 


\subsection{Sobrecarga docente y trabajo colaborativo}

Los análisis muestran que las docentes perciben una sobrecarga de tareas en su trabajo profesional. Esta sobrecarga muchas veces les genera emociones negativas como estrés, agotamiento, ansiedad y angustia. La superposición de actividades, el diseño y la evaluación de tareas personalizadas, la elaboración de diferentes materiales curriculares y la búsqueda de formas alternativas de "llegar al estudiantado" demandan muchas horas de trabajo, tal como señalan las participantes.

Grandes dificultades de todo tipo: tecnológicas, familiares, de tiempo, espacios, actividades laborales, actividades domésticas. Hubo momentos en los cuales el colapso emocional, físico e intelectual era próximo (...) muchísimas tareas, gestiones, reuniones, encuentros virtuales con docentes y con estudiantes al margen de las clases, etc. Eso fue y es muy agotador y complica demasiado toda la actividad docente. Fue y es muy cansando, agotador y por momentos, desequilibrante (Docente de nivel superior).

Las dificultades más relevantes son la regulación de los tiempos, los espacios en el hogar, y la simultaneidad de cuestiones familiares con lo laboral. En ocasiones se producen dificultades con la conexión a internet y otra dificultad es a veces los estados emocionales como desmotivación, angustia, ansiedad. Trato diariamente de organizarme a los fines de optimizar la tarea y también de aquietar la mente para favorecer la salud física y mental (Docente de nivel inicial).

Algunas docentes también perciben falta de motivación, tensión y agotamiento en el alumnado y sus familias, situación que adiciona complejidad a los procesos educativos remotos.

Se observa cierto desgaste y apatía de algunos estudiantes y familiares a continuar con la escolaridad de esta manera (Docente de nivel secundario).

Algunas familias están cansadas, yo no acompañan tanto a los chicos. También hay muchos problemas en las familias y por eso no pueden ayudarlos. (Docente de nivel primario).

Sin embargo, también hay docentes que señalan aspectos favorables y emociones positivas, especialmente vinculadas a la construcción de vínculos sólidos con estudiantes, familias y colegas. Algunas participantes sostienen que el trabajo colaborativo con otros docentes les ha permitido afrontar la compleja situación de enseñar en cuarentena. La 
construcción de redes es un aspecto que favorece el afrontamiento del estrés y el agotamiento que generan la sobrecarga de tareas y la necesidad de abordar múltiples problemáticas de manera simultánea.

Se ha intentado ir haciendo frente a las distintas variables trabajando en equipo con toda la institución, la directora y las compañeras han sido el pilar y entrecruzando estrategias, ideas hemos intentado y lo seguimos haciendo (Docente de nivel inicial)

Siempre se está realizando modificaciones, ya sea por la vulnerabilidad del alumno debido a la desigualdad de formas de comunicación como a los tiempos en que devuelven las actividades. Muchos alumnos en la ruralidad y sin conectividad. Además trabajo en red con los apoyos escolares de los municipios y los equipos de profesionales de la salud llámese psicóloga y psicopedagoga como así también las DAl de las escuelas especiales con quienes trabajamos codo a codo. (Docente de nivel primario y secundario)

Los resultados del presente estudio se relacionan con los hallados en otras investigaciones, Ardini et al. (2020) observaron que 98,2\% de las y los docentes encuestados manifiestan un incremento sustantivo en el tiempo destinado a la actividad laboral en pandemia. Este incremento está vinculado especialmente a la adecuación de las decisiones pedagógicas según los requerimientos de cada estudiante. También perciben sobrecarga laboral, situación que se complejiza aún más cuando no tienen apoyo institucional. Los resultados del estudio realizado por Bohé (2020) indican que el 60\% de los docentes de la institución escolar presentan sensaciones de agotamiento o cansancio emocional. La encuesta desarrollada por el Ministerio de Educación (2020) también muestra que la mayoría de las personas participantes señalaron que su trabajo aumentó considerablemente luego de la suspensión de clases presenciales.

Las docentes perciben que el incremento de horas de trabajo virtual y la superposición de actividades domésticas y de cuidado en el hogar les generan sensaciones de agotamiento, estrés y angustia. Asimismo, reconocen que el apoyo de otros docentes y la construcción de redes de trabajo colaborativo es un factor protector ante el agotamiento, el estrés y las múltiples dificultades, desiguales e incertidumbre que caracterizan al trabajo docente en el contexto actual. La Unesco (2020b) también señala problemas de estrés y confusión entre el profesorado, y enfatiza la importancia de construir estrategias de colaboración entre profesores, directivos y familias. Según el organismo, el apoyo psicosocial es indispensable 
en el marco de la emergencia educativa por COVID-19. Román et al. (2020) también observaron que la percepción de soporte emocional brindando y las habilidades socioemocionales se vinculan con la alta resiliencia de docentes durante la cuarentena.

Es necesario, en aislamiento y distanciamiento, pensar la docencia como un trabajo colaborativo en comunidades de aprendizaje. Es indispensable la construcción de espacios de diálogo, de experiencias y proyectos compartidos que integren al profesorado en un trabajo conjunto, solidario y comprometido. Estas comunidades de aprendizaje pueden contribuir a generar estrategias para afrontar el agotamiento, el estrés y las dificultades percibidas en el trabajo cotidiano.

También supone el trabajo con otros. La idea del docente como dueño del «tiempo» escolar en su propia «aula con muros», que ya venía erosionada, ha sido derribada por las circunstancias. Hace tiempo viene planteándose la necesidad de proyectos integrados de trabajo por equipos docentes. Se trata de la construcción de un profesionalismo colectivo que estudia, contextualiza y propone conjuntamente los desafíos cognitivos a los estudiantes. Esta idea de trabajo común se ve estimulada por la actual configuración de las sociedades en red, mediante conexiones rápidas de informaciones y posibilidades de trabajo remoto cooperativo (Dussel, Ferrante y Pulferp, 2020, p. 361)

Dussel et al. (2020) y Sabulsky (2020) también remarcan un emergente importante de la pandemia: el reconocimiento del trabajo docente por parte de diferentes sectores de la sociedad. La sociedad también parece revalorar la importancia de la escuela como espacio de encuentro y aprendizaje. "La escuela se extraña, se necesita, se revaloriza por parte de la sociedad (...) La escuela como espacio de lo común, como lugar del encuentro, del cuidado, de los lazos con las familias, entre generaciones, con el saber" (Sabulsky, 2020, p. 34)

Es indispensable apreciar el rol del profesorado y el esfuerzo realizado en el sostenimiento de la continuidad pedagógica en pandemia. El reconocimiento social deberá ir acompañado del económico, la problemática de salario y las condiciones laborales también deben ser discutidas.

\subsection{Especificidades de las propuestas según niveles educativos}

Los relatos de las docentes además de dar cuenta del trencadís educativo descrito en la primera categoría, muestran especificidades según los niveles educativos y edades del 
estudiantado. Las prácticas en contextos de educación inicial y primaria se caracterizan por estar mediadas por las familias. Es decir, los procesos educativos dependen en gran medida de la colaboración y disposición de la familia para poder desarrollarse. En estos casos, los familiares se constituyen en parejas pedagógicas, esta situación genera desigualdades entre el alumnado, ya que en algunos casos, la colaboración de la familia no es posible. En el caso de la educación inicial, se observan propuestas educativas que incluyen diversidad de objetos y recursos disponibles en el hogar. Asimismo, las docentes manifiestan desarrollar actividades lúdicas que incluyen a los miembros de la familia y a las mascotas. También dicen haber abordado en colaboración con los padres propuestas referidas a rutinas y usos de conocimientos en esas rutinas (numeración, escritura, etc.). Respecto de las mediaciones tecnologías, algunos manifiestan realizar encuentros sincrónicos grupales, aunque predominan las interacciones individuales o en grupos pequeños mediante videollamadas 0 audios de WhatsApp. En general, las evidencias de las tareas son enviadas por WhatsApp (fotos, audios y videos).

Los cambios que observo, es la necesidad de cambiar estrategias de enseñanza dirigida también a padres y tutores, siendo estos los transmisores de las propuestas. Otro es que sin el apoyo de la familia no se puede acceder al proceso del estudiante (Docente de nivel inicial).

Material manipulativo creado con reciclables, vídeos/programas pedagógicos, experimentos, fueron cambiando las propuestas (Docente de nivel primario).

No usé zoom porque no todos los chicos tienen conectividad...si empecé a hacer videollamadas para darles ánimo y fui con protocolo casa por casa repartiendo las actividades (Docente de nivel primario).

Las docentes de educación primaria también mencionan actividades que recuperan objetos del hogar y promueven la experimentación e investigación de fenómenos cotidianos. También aparece actividad vinculada a la preparación de alimentos, huertas y prácticas sustentables en el hogar. En la educación primaria también se propician tareas de búsqueda de información sobre problemáticas actuales y debates con las familias. Algunas docentes manifiestan realizar encuentros sincrónicos con estudiantes una o dos veces por semana. Las tareas son enviadas por WhatsApp o por correo electrónico y en algunos casos a través de plataformas. 
Las docentes de educación secundaria señalan diversidad de actividades de aprendizaje, entre las que se destacan búsqueda de información en diferentes fuentes, trabajos grupales, elaboración de videos y resolución de tareas disponibles en los materiales bibliográficos de cada asignatura. Los encuentros sincrónicos se realizan con más frecuencia que en la educación inicial y que en la primaria, generalmente a través de ZOOM o GOOGLE MEET. El uso de plataformas (EDMODO, CLASSROOM) para enviar y subir trabajos también es más frecuente que en el nivel inicial y primario.

Estrategias, recursos y actividades que utilicé fueron variadas de acuerdo a cada institución. Trabajo en tres colegios secundarios técnicos, con gestiones completamente distintas. Por ejemplo en una trabajamos ahora con la Metodología ABP (segmentada en equipos de docentes), en otra con actividades interdisciplinarias y en la otra con tareas propias de cada espacio curricular. Desde que comenzó la cuarentena, las actividades, los recursos y estrategias fueron naturalmente cambiando, de acuerdo a las respuestas de los estudiantes y de acuerdo a lo planteado por cada gestión (Docente de nivel secundario).

En el caso de la educación superior también se observan diversidad de propuestas pedagógicas. Si bien docentes y estudiantes parecen tener experiencias previas a la pandemia en el uso de plataformas educativas, en la cuarentena se masifica este uso y se adicionan clases virtuales y encuentros sincrónicos mediados por aplicaciones como JITSI, MEET o ZOOM. En la educación superior una problemática constante es la acreditación de los aprendizajes y las actividades de evaluación. Las profesoras han generado diferentes alternativas (exámenes orales, pruebas estandarizadas, tareas de desarrollo, elaboración de proyectos) para valorar los aprendizajes construidos por el estudiantado.

Lo que realizamos primero fue una reestructuración del programa y evaluamos qué material bibliográfico iba a continuar y cuáles otros iban a sugerirse como complementarios, como recursos empleamos guías de lectura donde hacíamos una presentación teórica, preguntas orientadoras, videos que complementaban el análisis. También realizamos presentaciones en PowerPoint o infografías complementadas con audios explicativos. Las actividades consistían en trabajos grupales de análisis de planificaciones, noticias actuales, entrevistas a docentes o reflexiones sobre cuestiones actuales, las cuales podían ser presentadas de diferentes maneras. $Y$ las evaluaciones tenían prácticamente la misma estructura de las actividades (Docente de nivel superior). 
Más allá de las especificidades de los niveles educativos, se observa en las expresiones de las docentes una preocupación constante por sostener las interacciones y el vínculo pedagógico. Ante esta preocupación se generan estrategias individualizadas para no perder el contacto con los estudiantes. La necesidad de la interacción, de verse, escucharse y hablarse, se pone en evidencia en las expresiones de las participantes. La pandemia visibiliza con claridad la necesidad del encuentro humano en los procesos educativos, por eso resulta importante para las docentes poder, de alguna manera, saber que los estudiantes están del otro lado de la pantalla. Los encuentros sincrónicos, si bien en muchos casos son difíciles de lograr por las dificultades mencionadas en categorías anteriores, son valorados por las docentes como oportunidades de interactuar con los estudiantes.

En síntesis, al igual que en trabajos previos (Birgin, 2020; Gómez et al. 2020; Macchiarola, Pizzolitto, Solivellas y Muñoz, 2020; Nuñez, 2020; Redondo, 2020) identificamos especificidades en las propuestas pedagógicas según el nivel educativo en el que se desempeñan las docentes. Los datos que hemos hallado también se relacionan con los resultados de la encuesta nacional a docentes realizada por el Ministerio de Educación de Argentina (2020), en el nivel inicial la propuesta más frecuente fue el envío de actividades para resolver en familia. En el nivel primario, las actividades fueron variadas, aunque predominaron aquellas de resolución individual, las acciones menos habituales fueron los intercambios orales y los encuentros sincrónicos. En secundaria, predominaron las tareas de resolución individual respecto de aquellas que requieren del intercambio y la coordinación de esfuerzos entre estudiantes. Las actividades a las que se enfrentaron mayormente las y los estudiantes supusieron respuestas de desarrollo breve.

Más allá de las especificidades de los niveles, las participantes manifiestan haber realizado adecuaciones a los programas de estudio y las planificaciones priorizando algunos contenidos educativos. La evaluación de los aprendizajes y el seguimiento de los estudiantes también es una preocupación constante de los docentes de todos los niveles. En este sentido, muchas instituciones han generado estrategias de valoración de los procesos educativos y algunos lineamientos en concordancia con las políticas educativas de cada provincia para la promoción del estudiantado. Dificultades, logros y desafíos específicos para cada nivel aparecen en las expresiones de las participantes Las docentes han generado variadas estrategias para sostener las interacciones y ofrecen posibilidades de aprendizaje a todos los estudiantes, considerando las particularidades de los niveles, las condiciones sociales de las familias y las adecuaciones curriculares realzadas. 


\subsection{Aprendizajes inesperados}

En el marco de las adversidades, dificultades y dificultades mencionadas, interesa subrayar un resultado importante que emerge de los análisis: las docentes identifican una serie de aprendizajes inesperados tanto en el estudiantado como en ellas mismas. Estos aprendizajes inesperados están vinculados especialmente a habilidades en el manejo, uso y apropiación de recursos tecnológicos y a la construcción de nuevos vínculos pedagógicos y novedosas formas de interacción, en algunos casos, más fluidas y personalizadas que en contextos presenciales. Asimismo, algunas docentes señalan aprendizajes no planificados referidos a conocimientos integrales, transversales e interdisciplinarios. También, mencionan procesos reflexivos que han favorecido la autorregulación, la flexibilidad y la apertura a diferentes alternativas de enseñanza y aprendizaje. Del mismo modo, las participantes reconocen procesos particulares de construcción de conocimientos actitudinales: los valores, la solidaridad y el trabajo colaborativo aparecen como los principales.

(...) aquellos que tienen que ver específicamente con actividades que se producen en la cotidianeidad del hogar: colaborar en la elaboración de comidas, poner la mesa, organizar los juguetes, hacer juegos en el patio de sus casas con materiales caseros, observar cómo funcionan los artefactos eléctricos que hay en el hogar, el compartir actividades con la familia (Docente de nivel inicial).

Hubo todo un aprendizaje en valores muy interesante, observé mucho compañerismo, solidaridad y empatía entre los estudiantes, con conciencia de lo que acontece en relación al cuidado y respeto por la salud de todos (...) También hubo todo un reconocimiento de la importancia de los otros, de la presencia, del contacto, del respeto a lo diferente y a las diferencias (Docente de nivel primario).

Aprendizajes que no estaban planificados fueron, por ejemplo, el empleo de herramientas digitales, donde todos debimos de ponernos a investigar sobre ello. $Y$ también el trabajo en grupo -tanto estudiantes como docentes- pero de manera virtual, a lo cual no estábamos acostumbrados (Docente de nivel primario).

Han aprendido a ser más autónomos, a organizar sus tiempos (Docente de nivel secundario)

Los aprendizajes inesperados ponen en evidencia los múltiples caminos que pueden tomar las propuestas educativas. Estos aprendizajes podrían considerarse experiencias educativas valiosas en la pandemia. Ardini et al. (2020) también hallaron este tipo de 
experiencias en las expresiones de docentes, entre las que se destacan la diversidad de maneras que hallaron para encontrarse con el alumnado, la cercanía e intensificación del vínculo, la posibilidad de trabajo colaborativo a través de la web y la capacidad creativa e innovadora para la presentación de temas o resolución de problemas en línea.

Un breve recorrido por buscadores de textos científicos y académico muestra numerosos relatos de experiencias de aprendizajes inesperados, resilientes y solidarios: diarios de pandemia (Martínez, 2020), microtalleres socio-emocionales virtuales (Muñoz, 2020), prácticas solidarias de grupos de madres (Tejero y Tejero, 2020), propuestas pedagógicas en las tranqueras (Redondo, 2020), lanchas llevando cuadernillos (Southwell, 2020) e interesantes memes sobre educación media (Nuñez, 2020), solo por mencionar algunos resultados de la búsqueda. Estas experiencias denotan procesos creativos que generan espacios novedosos para la enseñanza y el aprendizaje en contextos inciertos. Estos procesos creativos se caracterizan por generar acciones novedosas, nuevas formas de llegar al estudiantado, originales maneras de interactuar con otras personas y redes solidarias para intentar sostener el derecho a la educación. Los problemas (conectividad, acceso a los materiales, distancia, asilamiento, etc.) son resueltos buscando alternativas y activando pensamientos divergentes. Las acciones que emergen de estos pensamientos divergentes originan productos creativos y formas novedosas de resolución de problemas.

Cierta flexibilidad y divergencia puede observarse en las experiencias relatadas por las docentes, estas son características específicas de los contextos educativos creativos. Asimismo, la novedad y la sorpresa que definen muchas de las propuestas realizadas (experimentaciones, especialistas invitados, producciones audiovisuales, etc.), también definen los entornos creativos. Otros estudios también han señalado la importancia de las personas inesperadas como potenciales promotores de la creatividad en contextos educativos. Asimismo, las actividades que incluyen componentes novedosos y sorpresivos parecen ser propicias para la creatividad (Beghetto y Kaufman, 2011).

\section{Conclusiones}

Los análisis indican transformaciones en las prácticas docentes en diferentes momentos del aislamiento y distanciamiento. Cabe aclarar que las presentes conclusiones solamente son aplicables a la muestra de personas que participaron del estudio. La creación de propuestas alternativas y personalizadas es una constante en las expresiones de las participantes. Los relatos de las docentes dan cuenta de profundas desigualdades educativas, predominan las 
dificultades de conectividad, de disponibilidad de recursos tecnológicos y simbólicos para la educación remota y problemáticas referidas a la reorganización del tiempo y el espacio para actividades académicas en el hogar. También perciben sobrecarga en sus actividades profesionales e indican que el tiempo dedicado al trabajo ha aumentado considerablemente durante la pandémica. La necesidad de desarrollar propuestas personalizadas, entender situaciones particulares de los estudiantes y las familias e intentar promover procesos comunicativos fluidos demanda tiempo extra de trabajo.

Los relatos muestran ciertas especificidades en las propuestas según los niveles educativos y también algunas constantes: valorización de la comunicación y el sostenimiento del vínculo, priorización de contenidos curriculares y preocupación por el seguimiento y la evaluación de los estudiantes. Los datos nos han permitido identificar aprendizajes inesperados tanto en docentes como en estudiantes, estos aprendizajes se vinculan con la apropiación de recursos tecnológicos, habilidades para resolver problemas complejos y formas transversales, flexibles y abiertas de enseñar y aprender. Asimismo, se observan importantes aprendizajes actitudinales, procesos solidarios y acciones resilientes. Estos aprendizajes quizás no estaban planificados explícitamente al inicio del año escolar, sin embargo se han logrado. Es cierto que muchos contenidos planificados no se abordaron este año, no obstante es importante reconocer nuevos conocimientos relevantes que se han trabajado, en muchos casos con profundidad, en 2020.

Desde las teorías de la creatividad (Davies et al. 2013; Gajda et al. 2017; Richardson y Mishra, 2018), observamos que algunas de las propuestas pedagógicas relatadas por las docentes incluyen componentes que han sido definidos como propicios para la construcción de contextos creativos de enseñanza y aprendizaje: tareas que promueven la autonomía y la flexibilidad, propuestas que incluyen docentes inesperados y posibilidades de aprender en otros contextos (museos virtuales, conferencia, viajes, etc.), actividades de resolución de problemas, experimentación y búsqueda de respuestas alternativas, construcción de productos creativos (juegos, comidas, proyectos, investigaciones, recursos audiovisuales), utilización de multiplicidad de recursos educativos, construcción de redes colaborativas y asunción de los riesgos y desafíos que implica la enseñanza creativa. También son favorables para la creatividad los procesos de reflexión y autorregulación de las prácticas, la generación de cambios y transformación y la búsqueda de soluciones alternativas a los problemas. En la construcción de contextos creativos también se articula la agencia, el compromiso y la capacidad de resiliencia de las personas docentes, articulaciones que hemos observado en 
las narrativas. La metáfora del trencadís, como proceso creativo de construcción de alternativas educativas, ilustra el papel de la creatividad docente en tiempos de pandemia.

En el presente estudio nos hemos centrado en expresiones de las docentes que indican acciones creativas. No obstante, reconocemos que muchas propuestas educativas en tiempos de COVID-19 han limitado las posibilidades de aprendizaje al reproducir prácticas tradicionales y desconocer, las particularidades del alumnado y las desigualdades sociales. En futuros estudios es necesario profundizar en el análisis de este tipo de prácticas que no han favorecido los aprendizajes, sino que han profundizado las brechas educativas. Próximas investigaciones que amplíen las muestras y consideren las voces del estudiantado y las familias resultan indispensables para comprender el complejo fenómeno de la educación en pandemia.

El reducido número de participantes, la no inclusión de docentes varones en la muestra y la falta de estrategias de triangulación en la recolección de los datos son las principales limitaciones del estudio. Asimismo, es una limitación no considerar otras variables en el análisis, como contexto familiar, gestión institucional, condiciones socio-económicas estudiantiles, entre otras. Los análisis y los abordajes teóricos también resultan limitados para comprender una problemática tan compleja como la de las prácticas educativas en tiempos de pandemia. Se propone en futuros estudios ampliar los marcos teóricos referenciales articulando diversas categorías de análisis. El foco de análisis puesto en las prácticas creativas y los factores potenciadores de los aprendizajes ha limitado la lectura crítica de las expresiones de las docentes. Es interesante en futuros estudios ampliar la muestra de participantes e incluir las voces de las autoridades educativas, el estudiantado y sus familias. Asimismo, es relevante profundizar los análisis referidos las especificidades de los cambios educativos según niveles, contextos geográficos y socio-económicos. Considerar las particularidades de las instituciones educativas y las gestiones de los directivos también es una interesante línea de investigación. En próximos estudios también es interesante desarrollar análisis longitudinales de las transformaciones educativas durante y después de la pandemia.

Más allá de las limitaciones, consideramos que los análisis realizados contribuyen a la reflexión acerca de nuevos entornos educativos posibles. Mencionamos, a continuación, algunos posibles aportes del estudio al campo de la educación en tiempos de COVID-19. Para pensar la post-pandemia es indispensable valorar el trabajo realizado y el compromiso del profesorado en el sostenimiento de la continuidad educativa. Este reconocimiento comienza a hacerse visible en algunos sectores de la sociedad (Álvarez et al. 2020; Bocchio, 2020, Lion, 2020), queda pendiente el reconocimiento social y económico por parte de los gestores de las 
políticas educativas. Es necesaria también una reflexión crítica respecto de acciones de docentes, directivos y familias que han limitado el derecho a la educación. Es indispensable trabajar con estos grupos y generar acuerdos que garanticen aprendizajes y derechos del alumnado.

Dussel et al. (2020) nos invitan a pensar diferentes opciones para la educación en tiempos de pandemia y post-pandemia: educación de alternancia, educación bimodal, educación híbrida, educación aumentada y multimodalidad. Consideramos relevante aumentar las posibilidades de educación combinando diferentes estrategias, recursos y tecnologías dentro y fuera de las aulas y las instituciones educativas. Estas combinaciones no pueden ser yuxtaposiciones, sino procesos de integración entre disciplinas, contextos y contenidos que se articulen en objetivos educativos compartidos. Pensar en contextos de educación aumentada y multimodal en los que se integren diferentes prácticas comunicativas en espacios que trasciendan las aulas e integren a toda la comunidad parece un camino interesante para transitar en las nuevas normalidades. Los nuevos contextos educativos multimodales pueden recuperar experiencias de diferentes miembros de la comunidad y proyectos intergeneracionales mediados por artefactos tecnológicos actuales.

Las teorías de la creatividad en contextos educativos tienen interesantes aportes para la construcción de los contextos multimodales de enseñanza y aprendizaje. La originalidad, la flexibilidad, la apertura, el valor de las experiencias diversas, los contenidos indisciplinados (más allá de las disciplinas), las propuestas fuera de las aulas y las potencialidades de los diversos artefactos tecnológicos son condiciones identificadas como propicias para los contextos educativos creativos. Estas particularidades de los contextos creativos pueden ser de relevancia para discutir futuros entornos multimodales de educación en nuevas normalidades. Asimismo, recuperar y revalora las experiencias educativas construidas en pandemia es indispensable para imaginar nuevos contextos de enseñanza y aprendizaje. Reconocer el valor del trabajo realizado por docentes, estudiantes y familias es indispensable para pensar nuevas instituciones educativas.

\section{Agradecimiento}

Agradecemos el apoyo del Consejo Nacional de Investigaciones Científicas y Técnicas y la Universidad Nacional de Río Cuarto (Argentina). Proyecto de investigación: Desafíos actuales en creatividad (PIP 11220130100474). 


\section{Referencias}

Álvarez, Marisa., Gardyn, Natalia., lardelevsky, Alberto. y Rebello, Gabriel. (2020). Segregación Educativa en Tiempos de Pandemia: Balance de las Acciones Iniciales durante el Aislamiento Social por el Covid-19 en Argentina. Revista Internacional de Educación para la Justicia Social, 9(3). Recuperado de https://revistas.uam.es/riejs/article/view/12268

Ardini, Claudia., Herrera, María., González Angeletti, Valeria. y Secco, Nair. (2020). Docencia en tiempos de coronavirus: una mirada al trabajo docente y la experiencia educativa en entornos virtuales en el marco del ASPO por la pandemia COVID-19. Córdoba, Argentina: Mutual Conexión. Recuperado de https://rdu.unc.edu.ar/handle/11086/15887?show=full

Beghetto, Ronald. y Karwowski, Maciej. (2018). Educational consequences of creativity: A creative learning perspective. Creativity. Theories-Research-Applications, 5(2), 146154.Recuperado de https://content.sciendo.com/downloadpdf/journals/ctra/5/2/articlep146.xml

Beghetto, Ronald. y Kaufman, James. (2011). Teaching for creativity with disciplined improvisation. In R. Sawyer (Ed.), Structure and improvisation in creative teaching (pp. 94-110). Cambridge: Cambridge University Press. doi: http://dx.doi.org/10.1017/CBO9780511997105.006

Berg, Juliana., Blum Vestena, Carla. y Costa-Lobo, Cristina. (2020). Criatividade e Autonomia em Tempo de Pandemia: Ensaio Teórico a partir da Pedagogia Social. Revista Internacional de Educación para la Justicia Social, 9(3). Recuperado de https://revistas.uam.es/riejs/article/view/12180

Birgin, Alejandra. (2020). Emergencia y pedagogía: la cuarentena en los institutos de formación docente. En I. Dussel, P. Ferrante y D. Pulfer (Comp.), Pensar la educación en tiempos de pandemia: entre la emergencia, el compromiso y la espera (pp.189-200). Ciudad Autónoma de Buenos Aires: UNIPE Editorial Universitaria. Recuperado de http://biblioteca.clacso.edu.ar/Argentina/unipe/20200820015548/Pensar-laeducacion.pdf

Bocchio, María. (2020). El Futuro Llegó Hace Rato: Pandemia y Escolaridad Remota en Sectores Populares de Córdoba, Argentina. Revista Internacional De Educación Para La Justicia Social, 9(3). Recuperado de https://revistas.uam.es/riejs/article/view/12473

Bohé, Silvia. (2020). Indicadores de la presencia del síndrome de Burnout en docentes de una escuela secundaria rosarina durante el período de aislamiento/distanciamiento decretado a raíz de la pandemia COVID-19: propuesta de intervención para neutralizar dicha situación (Tesis de grado). Universidad Abierta Interamericana, Argentina.

Creswell, John. y Poth, Cheryl. (2007). Qualitative Inquiry and Research Design: Choosing among Five Approaches. Londres: Sage publications. 
Cristiano, Javier. (2018). Agencia, estructura y creatividad: tres modelos analíticos. Sociológica, 33(93), 119-150. Recuperado de http://www.scielo.org.mx/pdf/soc/v33n93/2007-8358-soc-33-93-119.pdf

Davies, Dan., Jindal-Snape, Divya., Collier, Chris., Digby, Rebecca., Hay, Penny. and Howe, Alan. (2013). Creative Learning Environments in Education. A Systematic Literature Review. Thinking Skills and Creativity, 8, 80-91. doi: https://doi.org/10.1016/j.tsc.2012.07.004

Dussel, Inés., Ferrante, Patricia. y Pulfer, Dario. (2020). Nuevas ecuaciones entre educación, sociedad, tecnología y Estado. En Inés Dussel, Patricia Ferrante y Darío Pulfer (Comp.), Pensar la educación en tiempos de pandemia: entre la emergencia, el compromiso y la espera (pp. 351-364). Ciudad Autónoma de Buenos Aires: UNIPE. Editorial Universitaria. Recuperado http://biblioteca.clacso.edu.ar/Argentina/unipe/20200820015548/Pensar-laeducacion.pdf

Expósito, Cristian. y Marsollier, Roxana. (2020). Virtualidad y educación en tiempos de COVID19. Un estudio empírico en Argentina. Educación y Humanismo, 22(39), 1-22. doi: https://doi.org/10.17081/eduhum.22.39.42141

Elisondo, Romina. (2015). La creatividad como perspectiva educativa. Cinco ideas para pensar los contextos creativos de enseñanza y aprendizaje. Actualidades Investigativas en Educación, 15(3), 566-588. Recuperado de https://www.scielo.sa.cr/scielo.php?script=sci arttext\&pid=S1409-47032015000300566

Elisondo, Romina., Melgar, María., Chesta, Rosana. y Siracusa, Marcela. (2020). Prácticas creativas en contextos educativos desiguales. Un estudio con docentes argentinos en tiempos de COVID-19. Diálogos sobre educación, 22, 1-19. Recuperado de http://www.dialogossobreeducacion.cucsh.udg.mx/index.php/DSE/article/view/873

Gajda, Aleksandra., Beghetto, Ronald. y Karwowski, Maciej. (2017). Exploring Creative Learning in the Classroom: A Multi-method Approach. Thinking Skills and Creativity, 24, 250-267. doi: https://doi.org/10.1016/j.tsc.2017.04.002

Glaveanu, Vlad., Hanchett Hanson, Michael., Baer, John., Barbot, Baptiste., Clapp, Edward., Corazza, Giovanni ... Sternberg, Robert. (2019). Advancing creativity theory and research: A sociocultural manifesto. Journal of Creative Behavior. Recuperado de https://onlinelibrary.wiley.com/doi/full/10.1002/jocb.395

Jeffrey, Bob. y Craft, Anna. (2004). Teaching creatively and teaching for creativity: distinctions and relationships. Educational studies, 30(1), 77-87. Recuperado de http://oro.open.ac.uk/425/

Lion, Carina. (2020). Enseñar y aprender en tiempos de pandemia: presente y horizontes Saberes y prácticas: Revista de Filosofía y Educación, 5(1), 1-8. Recuperado de http://revistas.uncu.edu.ar/ojs3/index.php/saberesypracticas/article/view/3675/2645 
Macchiarola, Viviana., Pizzolitto, Ana., Solivellas, Verónica. y Muñoz, Diego. (2020). La enseñanza con modalidad virtual en tiempos del COVID-19. La mirada de los estudiantes de la Universidad Nacional de Río Cuarto. Contextos de Educación, (28). Recuperado de http://www2.hum.unrc.edu.ar/ojs/index.php/contextos/article/view/1086/1149

Maldonado, Gabriel., Miró, María de los Ángeles., Stratta, Ana., Barreda, Antonio. y Zingaretti, Lara. (2020). La educación superior en tiempos de COVID-19: análisis comparativo México-Argentina. Revista de Investigación en Gestión Industrial, Ambiental, Seguridad y Salud en el Trabajo-GISST, 2(2), 35-60. Recuperado de https://www.editorialeidec.com/revista/index.php/GISST/article/view/79/60

Martínez, Sarah. (2020). Diarios jóvenes en una pandemia. Los registros de la Prepa Ibero Puebla. Revista Latinoamericana de Estudios Educativos, 50(ESPECIAL), 231-240. Recuperado de https://rlee.ibero.mx/index.php/rlee/article/view/107

Ministerio de Educación de Argentina. (2020). Informe Preliminar Encuesta a Docentes: Continuidad pedagógica en el marco del aislamiento por COVID-19. Recuperado de https://www.argentina.gob.ar/sites/default/files/informe preliminar encuesta a docente $\underline{\mathrm{s} \text { enpcp.pdf }}$

Muñoz, Verónica. (2020). Educación emocional mediante microtalleres durante clases virtuales en educación superior. En Sonia Brito, Lorena Basualto, Rodrigo Azócar, Victoria Gálvez y Claudia Flores (Eds.), Intervención social y educativa en tiempos de pandemia (pp. 17-30). Editorial Aún creemos en los sueños: Santiago de Chile. Recuperado

de https://repositorio.uautonoma.cl/bitstream/handle/20.500.12728/6923/Intervencio\%CC\%81n $\% 20$ social $\% 20$ y $\% 20$ educativa $\% 20$ en $\% 20$ tiempos $\% 20$ de $\% 20$ pandemia.pdf? sequence $=5 \&$ is Allowed $=y$

Nuñez, Pedro. (2020). Un tiempo escolar fuera de lo común: los jóvenes y sus sentidos sobre la escuela secundaria. En Inés Dussel, Patricia Ferrante y Darío Pulfer (Comp.), Pensar la educación en tiempos de pandemia: entre la emergencia, el compromiso y la espera (pp. 175-188). Ciudad Autónoma de Buenos Aires: UNIPE. Editorial Universitaria. Recuperado de http://biblioteca.clacso.edu.ar/Argentina/unipe/20200820015548/Pensar-laeducacion.pdf

Porta, Luis. (2015). Narrativas sobre la enseñanza en torno a la "didáctica de autor". Las maravillas cotidianas que abren a la percepción en el aula universitaria. Revista del IICE, (37), 41-54. doi: https://doi.org/10.34096/riice.n37.3449

Redondo, Patricia. (2020). Educar a la primera infancia en tiempos de excepción. En Inés Dussel, Patricia Ferrante y Darío Pulfer (Comp.), Pensar la educación en tiempos de pandemia: entre la emergencia, el compromiso y la espera (pp. 137-147). Ciudad Autónoma de Buenos Aires: UNIPE. Editorial Universitaria. Recuperado de http://biblioteca.clacso.edu.ar/Argentina/unipe/20200820015548/Pensar-laeducacion.pdf 
Richardson, Carmen. y Mishra, Punya. (2018). Learning Environments that Support Student Creativity: Developing the SCALE. Thinking Skills and Creativity, 27, 45-54. Recuperado de https://www.punyamishra.com/wp-content/uploads/2018/02/Richardson-Mishra-SCALE2017-pub.pdf

Román, Fabián., Forés, Anna., Calandri, Ismael., Gautreaux, Rosalba., Antúnez, Alejandro., Ordehi, Dalul., Barcelo, Ernesto ... Allegri, Ricardo. (2020). Resiliencia de docentes en distanciamiento social preventivo obligatorio durante la pandemia de COVID-19. Journal of Neuroeducation, 1(1), 76-87. Recuperado de https://revistes.ub.edu/index.php/joned/article/view/31727

Ruiz, Guillermo. (2020). Marcas de la Pandemia: El Derecho a la Educación Afectado. Revista Internacional De Educación Para La Justicia Social, 9(3), 45-59. Recuperado de https://revistas.uam.es/riejs/article/view/12355

Sabulsky, Gabriela. (2020). Del determinismo tecnológico al bricolaje digital. Sentidos y prácticas en tensión. Educación, Formación e Investigación, 6(10), 27-43.

Southwell, Myriam. (2020). Oficios terrestres, o del sostenimiento de la escolaridad entre virtualidad y territorio. En Inés Dussel, Patricia Ferrante y Darío Pulfer (Comp.), Pensar la educación en tiempos de pandemia: entre la emergencia, el compromiso y la espera (pp.163-173). Ciudad Autónoma de Buenos Aires: UNIPE. Editorial Universitaria. Recuperado de http://biblioteca.clacso.edu.ar/Argentina/unipe/20200820015548/Pensar-laeducacion.pdf

Tejero, Narja. y Tejero, Irán. (2020). Docencia y vínculos pedagógicos con la sociedad: experiencias de una comunidad durante la pandemia. Revista Latinoamericana de Estudios Educativos, 50(ESPECIAL), 263-270. doi: https://doi.org/10.48102/rlee.2020.50.ESPECIAL.111

Tranier, José., Bazán, Sonia., Porta, Luis. y Di Franco, María. (2020). Concatenaciones fronterizas: pedagogías, oportunidades, mundos sensibles y COVID-19. Praxis Educativa, 24(2), 1-18. Recuperado de https://cerac.unlpam.edu.ar/index.php/praxis/article/view/4812

UNESCO. (2020a). Impacto de la COVID-19 en la educación. Recuperado de https://es.unesco.org/covid19/educationresponse

UNESCO. (2020b). Enseñar en tiempos de COVID-19: una guía teórico-práctica para docentes. https://unesdoc.unesco.org/ark:/48223/pf0000373868?fbclid=lwAR26rU7YJHWm2Q N VJgC12Vf7-cyypr4h2D 07ndamWX8ooiKYa1DCKfnmY

UNICEF Argentina. (2020). Encuesta Covid-19. "Encuesta de Percepción y Actitudes de la Población. Impacto de la pandemia COVID-19 y las medidas adoptadas por el gobierno sobre la vida cotidiana". Recuperado de https://www.unicef.org/argentina/media/8056/ 


\section{Anexo 1 Cuestionario abierto Enseñar en cuarentena (construido para la AD-HOC para la presente investigación)}

En los primeros momentos del aislamiento social usted colaboró con nosotros respondiendo un cuestionario sobre enseñar en cuarentena. Han pasado cuatro meses desde aquel momento, ahora nos interesa conocer respecto de cambios en sus prácticas de enseñanza debido al aislamiento y el distanciamiento social por COVID-19. Nos gustaría que nos comentara sobre actividades nuevas que ha desarrollado y también respecto de obstáculos y dificultades que se le han presentado en estos últimos meses. Los datos recogidos serán tratados de manera confidencial. Puede colaborar con nosotros respondiendo este cuestionario o enviando las respuestas por audio. Agradecemos mucho su colaboración

Nombre:

Nivel educativo en el que trabaja:

1. ¿Qué cambios observa en su práctica como docente a partir del aislamiento y el distanciamiento social por COVID-19?

2. En los diferentes momentos del aislamiento y el distanciamiento, ¿fue realizando modificaciones en su práctica docente? ¿Por qué?

3. ¿Qué estrategias, recursos y actividades ha construido para enseñar y evaluar en este particular contexto? ¿Han ido cambiando en diferentes momentos de la cuarentena?

4. ¿Cómo caracterizaría su interacción con los alumnos y las familias (en el caso de estudiantes menores de edad) durante la cuarentena? ¿Observó cambios en diferentes momentos?

5. ¿Cuáles son las actividades más novedosas que ha propuesto durante el aislamiento y el distanciamiento?

6. ¿Qué dificultades se le presentaron para enseñar en estos tiempos? ¿Cómo afrontó estas dificultades? ¿Las dificultades fueron cambiando en diferentes momentos de la cuarentena?

7. ¿Considera que los estudiantes han construidos aprendizajes que no estaban planificados pero que son significativos para su formación? Si su respuesta es afirmativa, por favor cuéntenos que tipo de aprendizajes se han promovido en este particular contexto.

8. Los análisis preliminares del cuestionario administrado en abril indican que los docentes han desarrollado estrategias creativas para enseñar en cuarentena y dar continuidad a los procesos educativos ¿Qué opina al respecto?

9. Los análisis preliminares también muestran notorias desigualdades entre diferentes sectores sociales. ¿Qué opina al respecto? 


\section{Revista indizada en}
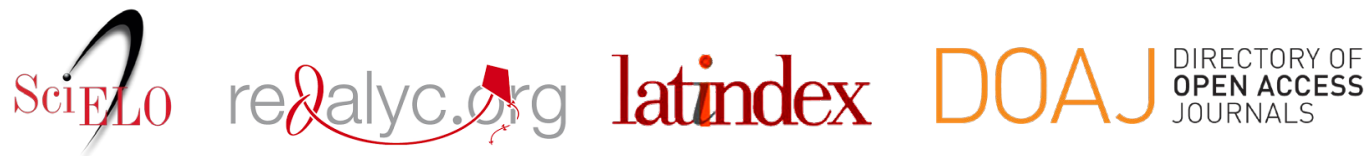

Distribuida en las bases de datos:
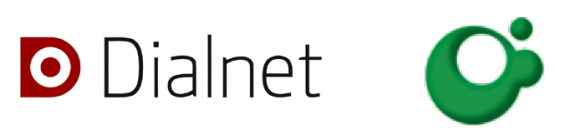
SHERPA/RøMEO

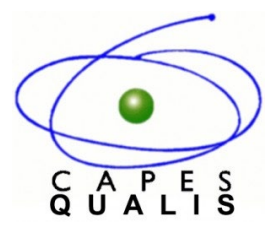

MIAR 